\title{
Fatores Associados ao Óbito Entre Pacientes Infectados Pelo Vírus Chikungunya, Pernambuco, Brasil (2015-2018)
}

\author{
Factors Associated with Death in Patients Infected by the Chikungunya Virus, \\ Pernambuco, Brazil (2015-2018)
}

\author{
Ana Cristina Pedrosa do Monte ${ }^{1}$ \\ Solange Laurentino dos Santos ${ }^{2}$
}

\section{RESUMO}

Objetivo: Analisar os fatores associados ao óbito entre pacientes com infecção pelo Vírus Chikungunya (CHIKV) e a distribuição espacial dos casos e dos óbitos confirmados no Estado de Pernambuco, Brasil. Metodologia: Estudo transversal, incluindo todos os casos e os óbitos registrados no Sistema de Informação de Agravos de Notificação, entre 2015 e 2018. Foram utilizados nas análises estatísticas os testes qui-quadrado de Pearson e Exato de Fisher, Odds ratio, intervalos de confiança de $95 \%$. As variáveis foram estudadas de modo univariado e com o modelo de regressão logística multivariado. A dependência espacial utilizou os índices bivariados Moran Global e o Local (Indicators of Spacial Association). Resultados: Homens (OR=2,1; IC 95\%: 1,5-3,0), com idade $\geq 60$ anos (OR=6,8; IC 95\%: 4,8-9,6) e moradores de zona urbana (OR=3,7; IC 95\%: 1,4-10,1), apresentaram maiores chances de evoluir para o óbito. As manifestações clínicas vômito (OR=1,8; IC 95\%: 1,2-2,6) e artrite (OR=2,0; IC 95\%: 1,3-3,0), comorbidades como diabetes (OR=9,7; IC 95\%: 5,3-17,5), hipertensão arterial (OR=9,2; IC 95\%: 5,8-14,4), doença renal crônica (OR=18,6; IC 95\%: 7,9-44,0) e hepatopatia (OR=6,0; IC 95\%: 1,9-19,3), mostraram-se associadas ao desfecho. As cidades de Recife, Olinda, Paulista, Camaragibe, Jaboatão dos Guararapes e Cabo de Santo Agostinho representaram agregados espaciais $(I=0,193 ; p<0,01)$ com alto número de casos e de óbitos confirmados. Conclusão: Os homens idosos apresentaram maiores chances de evolução para o óbito. $\mathrm{O}$ vômito e a artrite, além de comorbidades como a hipertensão arterial, o diabetes, a doença renal crônica e as hepatopatias foram condições associadas ao desfecho.

\section{DESCRITORES}

Febre de Chikungunya. Mortalidade. Epidemiologia.

\begin{abstract}
Objective: To analyze the factors associated with death among patients with CHIKV infection and the spatial distribution of confirmed cases and deaths in the state of Pernambuco, Brazil. Methodology: Cross-sectional study, including all cases and deaths confirmed by CHIKV infection in Pernambuco, Brazil, registered in the SINAN between 2015 and 2018. Pearson's chi-square and Fisher's exact tests were used, the association was estimated by the Odds ratio and $95 \%$ confidence intervals. The variables were studied in a univariate manner and with the multivariate logistic regression model. The spatial dependence used the bivariate Moran Global and local indexes (Indicators of Spacial Association). Results: Men (OR $=2.1 ; 95 \% \mathrm{Cl}: 1.5-3.0)$, aged $\geq 60$ years $(\mathrm{OR}=6.8 ; 95 \% \mathrm{Cl}: 4.8-9.6)$ and residents of an urban area $(\mathrm{OR}=3.7 ; 95 \% \mathrm{Cl}: 1.4-10.1)$, had a greater chance of progressing to death. Clinical manifestations of vomiting (OR $=1.8 ; 95 \% \mathrm{Cl}: 1.2-2.6)$ and arthritis $(\mathrm{OR}=2.0 ; 95 \% \mathrm{Cl}: 1.3-3.0)$, in addition to comorbidities such as diabetes $(\mathrm{OR}=9.7 ; 95 \% \mathrm{Cl}$ : 5.3-17.5), arterial hypertension (OR $=9.2 ; 95 \% \mathrm{Cl}: 5.8-14.4$ ), chronic kidney disease (OR $=18,6 ; 95 \% \mathrm{Cl}: 7.9-44.0)$ and liver disease $(\mathrm{OR}=6.0 ; 95 \% \mathrm{Cl}: 1.9-19.3)$ were associated with the outcome. In the state of Pernambuco, the cities of Recife, Olinda, Paulista, Camaragibe, Jaboatão dos Guararapes and Cabo de Santo Agostinho represented spatial aggregates (I $=0.193 ; \mathrm{p}<0.01$ ) with a high number of confirmed cases and deaths. Conclusion: Men and the elderly were more likely to die. Vomiting and arthritis were clinical conditions associated with the outcome, as well as the presence of comorbidities such as arterial hypertension, diabetes, chronic kidney disease and liver disease.
\end{abstract}

\section{DESCRIPTORS}

Chikungunya Fever. Mortality. Epidemiology.

\footnotetext{
${ }^{1}$ Mestranda em Saúde Coletiva pelo Programa de Pós-Graduação em Saúde Coletiva da Universidade Federal de Pernambuco, Recife, Pernambuco, Brasil.

${ }^{2}$ Professora Associada do Departamento de Medicina Social da Universidade Federal de Pernambuco, Recife, Pernambuco, Brasil.
} 
vírus chikungunya (CHIKV) é transmitido aos seres humanos por meio da picada de mosquitos infectados, principalmente, Aedes aegypti e Aedes albopictus ${ }^{1}$, além da via materno-fetal ${ }^{2}$.

Desde o reconhecimento da febre de chikungunya ( $\mathrm{CHIKF}$ ), casos esporádicos e grandes epidemias da doença por CHIKV foram relatadas na África ${ }^{3}$, Ásia e Pacífico ${ }^{4}$ e, mais recentemente, nas Américas ${ }^{5}$, representando um grave problema de saúde pública, sendo a segunda doença arboviral, transmitida pelo Aedes aegypti e Aedes albopictus, mais amplamente distribuída no mundo ${ }^{6}$.

Classicamente, a CHIKF se apresenta como uma doença febril, associada à artralgia, dor nas costas, dor de cabeça, fadiga e poliartralgia pronunciada afetando, principalmente, os tornozelos, os punhos, as falanges, os joeIhos e os cotovelos ${ }^{7-9}$. Nas condições crônicas, a poliartralgia ou poliartrite pode persistir como dor articular incapacitante e duradoura ${ }^{10}$, com sério impacto econômico e na qualidade de vida de indivíduos afetados ${ }^{11}$.

Historicamente, a doença não estava associada a altas taxas de mortalidade, mas nos últimos anos muitos estudos demonstraram a expressiva mortalidade associada ao CHIKV ${ }^{12-14}$, seja pelo agravamento de comorbidades pré-existentes ou por complicações causadas diretamente pela infecção pelo vírus chikungunya ${ }^{12}$.

Em 2014, os primeiros casos autóctones e a transmissão sustentada foram detectados no Brasil. Posteriormente, a rápida disseminação geográfica do vírus levou à grandes epidemias da doença por CHIKV por todos os estados brasileiros ${ }^{15}$. Contribuíram para a emergência, disseminação e epide- mias do CHIKV fatores como a alta dispersão dos vetores ${ }^{6}$, circulação simultânea do Vírus da Dengue (DENV), Vírus Zika (ZIKV) e o $\mathrm{CHIKV}^{14}$, população suscetível ${ }^{16}$, além de condições socioeconômicas e demográficas desfavoráveis ${ }^{17}$.

O Estado de Pernambuco, no ano de 2016, foi o mais afetado pela epidemia da doença no Brasil ${ }^{14}$. Não está claro se epidemias de grandes magnitudes ocorrerão nos anos subsequentes, mas considerando que a região apresenta condições para a disseminação e manutenção do CHIKV é possível que ocorram.

A possibilidade de tornar-se uma doença endêmica, juntamente com outros arbovírus é particularmente preocupante. Portanto, é imperativo estudar o comportamento espacial, epidemiológico e clínico, a fim de aumentar os esforços para a vigilância e o diagnóstico precoce.

O objetivo principal do estudo foi analisar os fatores associados ao óbito entre pacientes com infecção pelo CHIKV e a distribuição espacial de casos e óbitos confirmados nos municípios do Estado de Pernambuco.

\section{METODOLOGIA}

Foi realizado um estudo transversal, incluindo todos os casos prováveis (pacientes confirmados e que aguardam confirmação) e óbitos confirmados por infecção pelo CHIKV, no Estado do Pernambuco, Nordeste do Brasil, entre 2015 e 2018. Os dados foram coletados dos relatórios de notificação do Sistema de Informação de Agravos de Notificação (SINAN), da Secretaria Estadual de Saúde de Pernambuco. 
A confirmação da infecção pelo CHIKV seguiu as diretrizes do Ministério da Saúde do Brasil ${ }^{16,18}$, baseada no resultado reagente/positivo para os exames laboratoriais: pesquisa de genoma do CHIKV por Reação em Cadeia da Polimerase via Transcriptase Reversa (RT-PCR), detecção de anticorpos Imunoglobulina M (IgM) por testes sorológicos (Ensaio Imunoenzimático - ELISA), exame anatomopatológico com pesquisa de antígenos virais por imuno-histoquímica, além do critério clínico-epidemiológico que consiste na confirmação de casos por vínculo epidemiológico, após a confirmação laboratorial dos primeiros casos de uma área.

A confirmação ou o descarte da CHIKF como a causa básica do óbito depende de minuciosa investigação domiciliar, buscas em prontuários hospitalares e de informações epidemiológicas. Todos esses dados subsidiam a discussão no Comitê de Discussão de Óbitos por Arboviroses ${ }^{18}$. Todos os óbitos confirmados incluídos na pesquisa foram discutidos pelo comitê.

Comparamos os pacientes que tiveram a doença e evoluíram para cura (sobreviventes) e os que evoluíram para o óbito (não sobreviventes), a fim de investigar os fatores associados à morte por infecção pelo CHIKV.

Foram estudadas variáveis socioeconômicas e demográficas (sexo, idade, raça/ cor, escolaridade e zona de residência), relacionadas às manifestações clínicas (vômito, artrite, cefaleia, náuseas, dor nas costas, artralgia, febre, mialgia, exantemas e leucopenia) e comorbidades (diabetes, hipertensão arterial, doença renal crônica e hepatopatias), além do município de residência dos pacientes.
As análises estatísticas foram realizadas no programa Epilnfo ${ }^{\mathrm{TM}}$ versão 7.2.2.6. Os testes Qui-quadrado de Pearson e Exato de Fisher foram utilizados. A magnitude da associação foi estimada pela razão de chances (Odds Ratio, OR), com intervalo de confiança (IC) de 95\%.

O estudo dos dados utilizou dois modelos explicativos: um com os fatores independentes, estudados de modo bivariado, sendo a relação entre exposição e desfecho analisada isoladamente; e o modelo de regressão logística multivariado incluindo todos os fatores que apresentaram significância estatística $(p \leq 0,05)$ na primeira análise.

A distribuição espacial foi realizada nos softwares GeoDa 1.14.0 e QGis versão 2.14.1 Essen, sistema de projeção Universal Transversa de Mercator (UTM) e Datum Sistema de Referência Geocêntrico para as Américas (SIRGAS) 2000. A dependência espacial foi estudada utilizando os Índices bivariado Moran Global e o bivariado Local de Moran (Local Indicators of Spatial Association - Lisa). Foram verificadas a existência de associação espacial linear entre o número de casos e óbitos por febre de chikungunya em Pernambuco, considerando a significância $p<0,05$.

A distribuição espacial do número médio de casos prováveis e óbitos confirmados entre os municípios foi demonstrada através de dois mapas temáticos estratificados por quartil. Para a visualização de dependência espacial foi utilizado Moran Map, que apresenta os agregados com significância estatística $(p<0,05)$, segundo o diagrama de espalhamento de Moran.

Trata-se de comparar o valor de cada município para uma variável com a média dos 
seus vizinhos para a outra variável, da seguinte forma: no quadrante 1 (alto-alto) estão as unidades espaciais que exibiram valores altos da variável de interesse (número médio de casos prováveis) e são rodeados por unidades espaciais que apresentam valores também altos para a outra variável (número de óbitos confirmados); no quadrante 2 (baixo-baixo) estão as unidades espaciais que exibiram valores baixos da variável de interesse e são rodeados por unidades espaciais que apresentam valores também baixos para a outra variável; no quadrante 3 (alto-baixo) estão as unidades espaciais que exibiram valores altos da variável de interesse e são rodeados por unidades espaciais que apresentam valores baixos para a outra variável; no quadrante 4 (baixo-alto) estão as unidades espaciais que exibiram valores baixos da variável de interesse e são rodeados por unidades espaciais que apresentam valores altos para a outra variável.

O protocolo de pesquisa foi aprovado pelo Comitê de Ética em Pesquisa da Universidade Federal de Pernambuco CEP/UFPE (número do CAAE 19062419.0.0000.5208) com anuência da Secretaria Estadual de Saúde de Pernambuco (SES-PE).

\section{RESULTADOS}

Foram registrados 57.459 casos prováveis e 128 óbitos confirmados para febre de chikungunya no Estado de Pernambuco, Brasil, no período de 2015 a 2018. A incidência média e a letalidade foram de 152,9 casos/ 100 mil habitantes e 0,9\%, respectivamente.

A análise univariada dos dados demográficos apontou que a maioria dos sobreviventes era do sexo feminino $(61,8 \%)$, com idade média de $36 \pm 21$ anos, se contrapondo aos não sobreviventes que eram do sexo masculino $(56,3 \%)$, com idade média de $55 \pm 29$ anos (Tabela 1).

Entre os não sobreviventes, homens (OR=2,1; IC 95\%: 1,5-3,0), com idade maior ou igual a 60 anos (OR=6,8; IC 95\%: 4,8-9,6) e moradores de zona urbana $(\mathrm{OR}=3,7$; IC 95\%: 1,4-10,1) apresentaram maiores chances de óbito. As variáveis raça/cor de pele e escolaridade não apresentaram significância estatística quanto a possíveis associações com o desfecho (Tabela 1).

As manifestações clínicas com maiores chances para o desfecho entre os não sobreviventes foram vômito (OR=1,8; IC 95\%: $1,2-2,6)$ e artrite (OR=2,0; IC 95\%: 1,3-3,0), estando associados a menores chances de óbito a presença de cefaleia (OR=0,5; IC 95\%: $0,3-0,7)$, náuseas (OR=0,4; IC 95\%: 0,2-0,7), dor nas costas (OR=0,6; IC 95\%: 0,3-0,9) e artralgia (OR=0,6; IC 95\%: 0,4-0,9). Sinais e sintomas como febre, mialgia, exantemas e leucopenia não apresentaram significância estatística quanto a possíveis associações com o desfecho (Tabela 2).

Entre as comorbidades pré-existentes, mostraram-se associadas a maiores chances para o desfecho a presença de diabetes (OR=9,7; IC 95\%: 5,3-17,5), hipertensão arterial (OR=9,2; IC 95\%: 5,8-14,4), doença renal crônica (OR=18,6; IC 95\%: 7,9-44,0) e hepatopatia (OR=6,0; IC 95\%: 1,9-19,3) (Tabela 3).

$\mathrm{Na}$ análise multivariada, mantiveram-se com maiores chances de óbito o sexo masculino (60\%), idade maior ou igual a 60 anos $(30 \%)$, presença das manifestações clínicas vômito (80\%) e artrite (50\%) e entre 
Tabela 1. Fatores socioeconômicos e demográficos associados a pacientes com infecção pelo vírus chikungunya, comparação entre sobreviventes e não sobreviventes, Pernambuco, Brasil, 2015-2018.

\begin{tabular}{|c|c|c|c|c|c|c|}
\hline \multirow[t]{2}{*}{ Variáveis* } & \multirow{2}{*}{$\begin{array}{l}\text { Sobreviventes } \\
\quad(n=57.459)\end{array}$} & \multicolumn{2}{|c|}{$\begin{array}{l}\text { Não sobreviventes } \\
\qquad(\mathrm{n}=128)\end{array}$} & \multirow[t]{2}{*}{ OR } & \multirow[t]{2}{*}{ IC 95\% } & \multirow[t]{2}{*}{ p-valor } \\
\hline & & $\mathrm{n}$ & $\%$ & & & \\
\hline Sexo & & & & & & $<0.0000$ \\
\hline Feminino & 35.518 & 56 & 0.2 & 01 & - & \\
\hline Masculino & 21.932 & 72 & 0.3 & 2.1 & $1.5-3.0$ & \\
\hline Idade & (36 \pm 21 anos) & \multicolumn{2}{|c|}{ (55 \pm 29 anos) } & & & 0.0000 \\
\hline$<60$ anos & 48.978 & 59 & 0.1 & 01 & - & \\
\hline$\geq 60$ anos & 8.479 & 69 & 0.8 & 6.8 & $4.8-9.6$ & \\
\hline Raça/cor & & & & & & 0.0636 \\
\hline Branco & 4.910 & 16 & 0.3 & 01 & - & \\
\hline Pardo ou Preto & 22.587 & 43 & 0.2 & 0.6 & $0.3-1.0$ & \\
\hline Escolaridade & & & & & & 0.1471 \\
\hline \multicolumn{7}{|l|}{ (anos de estudo) } \\
\hline$<8$ anos & 5.604 & 11 & 0.2 & 0.5 & $0.2-1.3$ & \\
\hline$\geq 8$ anos & 5.468 & 5 & 0.1 & 01 & - & \\
\hline Zona de residência & & & & & & 0.0033 \\
\hline Rural & 6.199 & 4 & 0.1 & 01 & - & \\
\hline Urbana & 46.302 & 111 & 0.2 & 3.7 & $1.4-10.1$ & \\
\hline
\end{tabular}

*Totais variam devido à exclusão de casos em branco e ignorados; (Média \pm Desvio padrão); OR: Odds Ratio; IC: Intervalo de Confiança; Teste Qui-quadrado de Pearson e Exato de Fisher; nível de significância p<0,05.

as comorbidades pré-existentes doença renal crônica (90\%) e hipertensão arterial (40\%). Com exceção das comorbidades, as demais variáveis tiveram um efeito aumentado em relação à análise univariada (Tabela 4).

A análise da distribuição espacial dos casos prováveis e dos óbitos confirmados nos municípios pernambucanos, demonstrou associação espacial positiva e significativa através do índice bivariado Moran Global $(I=0,193 ; p<0,01)$.

O Moran Map bivariado evidenciou seis clusters do tipo Alto-Alto, nos municípios de Recife, Olinda, Paulista, Camaragibe, Jaboatão dos Guararapes e Cabo de Santo
Agostinho. Nessas regiões ocorreram elevados quantitativos de casos e óbitos, indicando que as áreas que apresentaram alto número de casos possuem cidades vizinhas com alto número de óbitos (Figura 1).

\section{DISCUSSÃO}

A letalidade geral atribuída à infecção pelo $\mathrm{CHIKV}$ foi menor que $1 \%$, semelhante ao observado em análises realizadas no Estado do Ceará, Nordeste do Brasili,9 e divergente de estudos que demonstraram uma mortalidade importante associada ao CHIKV 5,12-14,19.

Sobre as mortes por febre de chikun- 
Tabela 2. Sinais e sintomas associados a pacientes com infecção pelo vírus chikungunya, comparação entre sobreviventes e não sobreviventes, Pernambuco, Brasil, 2015-2018.

\begin{tabular}{|c|c|c|c|c|c|c|}
\hline \multirow[t]{2}{*}{ Variáveis* } & \multirow{2}{*}{$\begin{array}{l}\text { Sobreviventes } \\
\quad(n=57.459)\end{array}$} & \multicolumn{2}{|c|}{$\begin{array}{c}\text { Não sobreviventes } \\
\qquad(n=128)\end{array}$} & \multirow[t]{2}{*}{ OR } & \multirow[t]{2}{*}{ IC 95\% } & \multirow[t]{2}{*}{$\mathrm{p}$-valor } \\
\hline & & $n$ & $\%$ & & & \\
\hline Vômito & & & & & & 0.0057 \\
\hline Ausente & 17.318 & 83 & 0.5 & 01 & - & \\
\hline Presente & 3.919 & 33 & 0.8 & 1.8 & $1.2-2.6$ & \\
\hline Artrite & & & & & & 0.0015 \\
\hline Ausente & 18.281 & 88 & 0.5 & 01 & - & \\
\hline Presente & 2.956 & 28 & 0.9 & 2.0 & $1.3-3.0$ & \\
\hline Cefaleia & & & & & & 0.0000 \\
\hline Ausente & 9.157 & 72 & 0.8 & 01 & - & \\
\hline Presente & 12.080 & 44 & 0.4 & 0.5 & $0.3-0.7$ & \\
\hline Náuseas & & & & & & 0.0023 \\
\hline Ausente & 16.995 & 106 & 0.6 & 01 & - & \\
\hline Presente & 4.242 & 10 & 0.2 & 0.4 & $0.2-0.7$ & \\
\hline Dor nas costas & & & & & & 0.0426 \\
\hline Ausente & 17.300 & 103 & 0.6 & 01 & - & \\
\hline Presente & 3.937 & 13 & 0.3 & 0.6 & $0.3-0.9$ & \\
\hline Artralgia & & & & & & 0.0055 \\
\hline Ausente & 5.793 & 45 & 0.8 & 01 & - & \\
\hline Presente & 15.444 & 71 & 0.5 & 0.6 & $0.4-0.9$ & \\
\hline Febre & & & & & & 0.8800 \\
\hline Ausente & 2.292 & 13 & 0.6 & 01 & - & \\
\hline Presente & 18.945 & 103 & 0.5 & 01 & $1.0-1.7$ & \\
\hline Mialgia & & & & & & 0.7700 \\
\hline Ausente & 8.894 & 47 & 0.5 & 01 & - & \\
\hline Presente & 12.343 & 69 & 0.6 & 1.1 & $0.7-1.5$ & \\
\hline Exantema & & & & & & 0.3923 \\
\hline Ausente & 14.162 & 73 & 0.5 & 01 & - & \\
\hline Presente & 7.075 & 43 & 0.6 & 1.2 & $0.8-1.7$ & \\
\hline Leucopenia & & & & & & 0.4577 \\
\hline Ausente & 20.869 & 113 & 0.5 & 01 & - & \\
\hline Presente & 368 & 03 & 0.8 & 1.5 & $0.5-4.8$ & \\
\hline
\end{tabular}

*Totais variam devido à exclusão de casos em branco e ignorados; OR: Odds Ratio; IC: Intervalo de Confiança; Teste Qui-quadrado de Pearson e Exato de Fisher; nível de significância p<0,05. 
Tabela 3. Comorbidades associadas a pacientes com infecção pelo vírus chikungunya, comparação entre sobreviventes e não sobreviventes, Pernambuco, Brasil, 2015-2018.

\begin{tabular}{|c|c|c|c|c|c|c|}
\hline \multirow[t]{2}{*}{ Variáveis* } & \multirow{2}{*}{$\begin{array}{l}\text { Sobreviventes } \\
\quad(n=57.459)\end{array}$} & \multicolumn{2}{|c|}{$\begin{array}{c}\text { Não sobreviventes } \\
\quad(n=128)\end{array}$} & \multirow{2}{*}{ OR } & \multirow{2}{*}{ IC 95\% } & \multirow{2}{*}{ p-valor } \\
\hline & & $n$ & $\%$ & & & \\
\hline Diabetes & & & & & & 0.0000 \\
\hline Ausente & 20.965 & 103 & 0.5 & & & \\
\hline Presente & 272 & 13 & 4.6 & 9.7 & $5.34-17.5$ & \\
\hline Hipertensão arterial & & & & & & 0.0000 \\
\hline Ausente & 20.619 & 91 & 0.4 & & & \\
\hline Presente & 618 & 25 & 3.9 & 9.2 & $5.8-14.4$ & \\
\hline Doença Renal Crônica & & & & & & 0.0000 \\
\hline Ausente & 21.175 & 110 & 0.5 & & & \\
\hline Presente & 62 & 06 & 8.8 & 18.6 & $7.9-44.0$ & \\
\hline Hepatopatia & & & & & & 0.0015 \\
\hline Ausente & 21.144 & 113 & 0.5 & & & \\
\hline Presente & 93 & 03 & 3.1 & 6.0 & $1.9-19.3$ & \\
\hline
\end{tabular}

*Totais variam devido à exclusão de casos em branco e ignorados; OR: Odds Ratio; IC: Intervalo de Confiança;

Teste Qui-quadrado de Pearson e Exato de Fisher; nível de significância $p<0,05$.

Tabela 3. Comorbidades associadas a pacientes com infecção pelo vírus chikungunya, comparação entre sobreviventes e não sobreviventes, Pernambuco, Brasil, 2015-2018.

\begin{tabular}{|c|c|c|c|c|c|c|}
\hline \multirow[t]{2}{*}{ Variáveis* } & \multirow{2}{*}{$\begin{array}{l}\text { Sobreviventes } \\
(n=57.459)\end{array}$} & \multicolumn{2}{|c|}{$\begin{array}{l}\text { Não sobreviventes } \\
\qquad(n=128)\end{array}$} & \multirow{2}{*}{ OR } & \multirow{2}{*}{ IC 95\% } & \multirow{2}{*}{$\mathrm{p}$-valor } \\
\hline & & $\mathrm{n}$ & $\%$ & & & \\
\hline Diabetes & & & & & & 0.0000 \\
\hline Ausente & 20.965 & 103 & 0.5 & & & \\
\hline Presente & 272 & 13 & 4.6 & 9.7 & $5.34-17.5$ & \\
\hline Hipertensão arterial & & & & & & 0.0000 \\
\hline Ausente & 20.619 & 91 & 0.4 & & & \\
\hline Presente & 618 & 25 & 3.9 & 9.2 & $5.8-14.4$ & \\
\hline Doença Renal Crônica & & & & & & 0.0000 \\
\hline Ausente & 21.175 & 110 & 0.5 & & & \\
\hline Presente & 62 & 06 & 8.8 & 18.6 & $7.9-44.0$ & \\
\hline Hepatopatia & & & & & & 0.0015 \\
\hline Ausente & 21.144 & 113 & 0.5 & & & \\
\hline Presente & 93 & 03 & 3.1 & 6.0 & $1.9-19.3$ & \\
\hline
\end{tabular}

*Totais variam devido à exclusão de casos em branco e ignorados; OR: Odds Ratio; IC: Intervalo de Confiança;

Teste Qui-quadrado de Pearson e Exato de Fisher; nível de significância $p<0,05$. 
Tabela 4. Fatores associados (regressão logística multivariada) a mortes em pacientes com infecção pelo vírus chikungunya, Pernambuco, Brasil, 2015-2018.

\begin{tabular}{lccc}
\multicolumn{1}{c}{ Fator } & OR & IC 95\% & p-valor \\
\hline Sociodemográfico & & & \\
$\quad$ Sexo (masculino) & 2.6 & $1.73-3.85$ & 0.0000 \\
$\quad$ Idade ( $\geq 60$ anos) & 7.3 & $4.82-11.08$ & 0.0000 \\
Sinais e Sintomas & & & \\
$\quad$ Vômito & 2.8 & $1.81-4.41$ & 0.0000 \\
$\quad$ Artrite & 2.5 & $1.48-4.06$ & 0.0005 \\
Comorbidades & & & \\
$\quad$ Doença Renal Crônica & 5.9 & $1.84-18.82$ & 0.0029 \\
$\quad$ Hipertensão Arterial & 4.4 & $2.36-8.32$ & 0.0000 \\
\hline
\end{tabular}

OR: Odds Ratio; IC: Intervalo de Confiança; Teste Qui-quadrado de Pearson e Exato de Fisher; nível de significância $p<0,05$.

Figura 1. Distribuição espacial dos casos prováveis e óbitos confirmados para febre de chikungunya: casos por quartis (A), óbitos por quartis (B) e índice bivariado de Moran (Moran Map, C), Pernambuco, Brasil, 2015 - 2018.

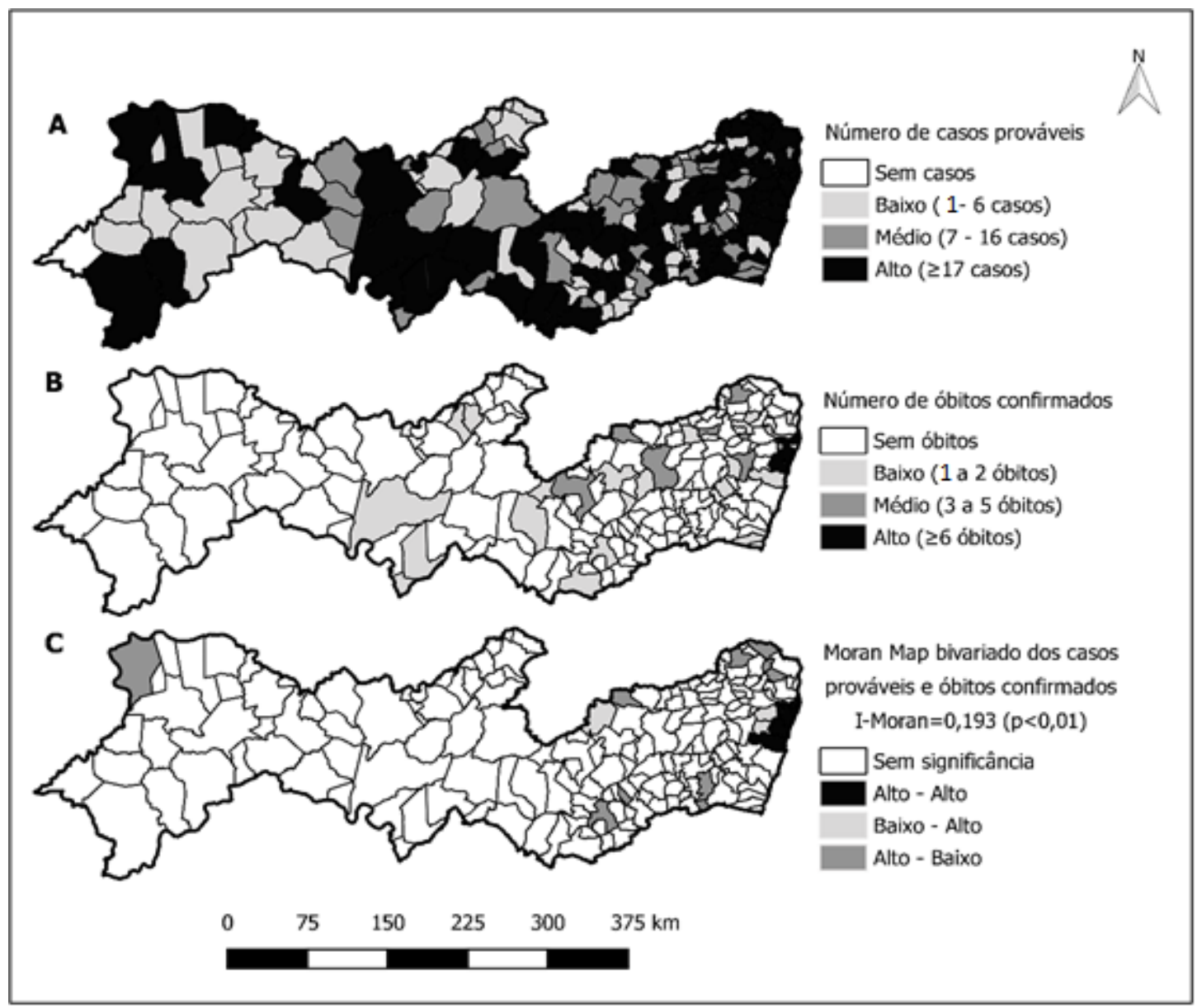

Fonte: próprio autor. 
gunya ocorridas em Pernambuco durante a epidemia de 2016, estudos apontaram um aumento médio de 4.370 mortes em comparação com a média de anos anteriores e mortalidade de 47,9 óbitos por 100.000 habitantes, sendo o estado mais afetado no Brasil pela epidemia da doença em 2016 ${ }^{12,14}$.

A literatura indica que o sistema passivo de Vigilância Epidemiológica utilizado no Brasil detectou um número, aproximadamente, 50 vezes menor de mortes por CHIKF em Pernambuco ${ }^{12,14}$. Nem todas essas mortes estimadas podem ser causadas por complicações da doença, mas podem ter sido motivadas pelo agravamento de comorbidades pré-existentes ou complicações causadas diretamente pela infecção do CHIKV 12,14,19.

As características sociodemográficas dos grupos estudados evidenciaram que os sobreviventes eram em sua maioria do sexo feminino, com idade menor que 60 anos e moradoras de zona urbana, semelhante ao descrito em outros trabalhos ${ }^{8,9}$. Entre os não sobreviventes, observamos que pacientes homens, com idade de 60 anos ou mais e moradores de zona urbana, apresentaram maior risco de morte, consistindo com os achados de surtos anteriores em que o aumento da idade estava associado a óbitos ${ }^{5,8,9,19,20}$.

Quanto as manifestações clínicas, a presença de vômito e de artrite se mostraram associadas a maiores chances de óbitos, tanto na análise univariada quanto na multivariada, compatível com a literatura ${ }^{8,9,20,22}$.

Em estudo realizado nas Antilhas Francesas, apenas $8,2 \%$ dos pacientes idosos com CHIKF tinham uma apresentação clínica clássica e $32 \%$ não apresentavam artralgia ${ }^{21}$. Esses aspectos podem atrasar medidas de apoio que possam impedir mortes, dificultar o diagnóstico clínico e, como consequência, levar à subnotificação de mortes pela doença ${ }^{19}$.

Outros sinais e sintomas como cefaleia, náuseas, dor nas costas e artralgia se apresentaram associados com menores chances de óbito entre pacientes infectados pelo $\mathrm{CHIKV}^{8,9}$. Uma explicação para isso pode ser o fato de que, ao manifestar esses sintomas, os pacientes procuram atendimento médico ou as equipes de saúde fornecem tratamento mais cuidadoso ${ }^{9}$.

Os resultados sugerem associação importante entre o óbito por CHIKV e comorbidades como diabetes, hipertensão arterial, doença renal crônica e hepatopatia, tal como em outros trabalhos com comorbidades como diabetes, doenças hepáticas, doenças neurológicas, hipertensão ou função renal comprometida geralmente desenvolvem infecção grave por $\mathrm{CHIKV}^{23}$. Pesquisas realizadas nas Ihas da Martinica e Guadalupe, demonstraram 74 mortes associadas ao $\mathrm{CHIKV}$, sendo $85 \%$ apresentaram pelo menos uma comorbidade, como diabetes $(30 \%)$, doenças renais $(55,4 \%)$ e hepáticas $(24,3 \%)^{20}$.

Estudos recentes evidenciaram que pacientes com hipertensão arterial, diabetes e Doença Renal Crônica (DRC) infectados com CHIKV apresentaram sintomas mais graves e prolongados da doença, necessitando de hospitalização e doses aumentadas de medicamentos ${ }^{9,25,26}$.

Em pacientes diabéticos, a infecção pelo CHIKV altera os níveis de glicose e piora significativamente os sintomas, desenvolvendo complicações agudas, o que implica maior morbidade nesses pacientes ${ }^{26}$. A presença de 
DRC aumenta a frequência de manifestações clínicas mais graves, devido ao decréscimo na função imune ${ }^{9}$.

A análise da distribuição espacial dos casos prováveis e dos óbitos confirmados nos municípios pernambucanos, demonstrou que as cidades de Recife, Olinda, Paulista, Camaragibe, Jaboatão dos Guararapes e Cabo de Santo Agostinho representam agregados com alto número de casos e óbitos $(I=0,193$; $p<0,01$ ),

A maioria das doenças transmissíveis, incluindo a febre de chikungunya, são afetadas por contextos que envolvem habitações precárias e superlotadas, alta densidade populacional, baixa escolaridade, desemprego, baixo Índice de Desenvolvimento Humano Municipal e problemas de cobertura na atenção à saú$\mathrm{de}^{17,27-29}$. O fornecimento ineficiente de água, serviços de coleta de lixo e saneamento inadequado, falta de medidas preventivas ${ }^{29}$ e a proximidade geográfica ${ }^{30}$ também favorecem a ocorrência de surtos da doença. Torna-se importante o conhecimento da distribuição espacial dos determinantes socioeconômicos

\section{REFERÊNCIAS}

1. Powell JR. Perspective piece mosquito-borne human viral diseases: Why aedes aegypti? Am J Trop Med Hyg. 2018; 98(6):1563-1565.

2. Lyra P, Campos G, Bandeira I, Sardi S, Costa L, Santos $F$, et al. Congenital Chikungunya Virus Infection after an Outbreak in Salvador, Bahia, Brazil. Am J Perinatol Reports. 2016 Aug 22; 06(03):e299-300. e ambientais, dessa forma, sugerimos novos estudos abordando tais questões.

\section{CONCLUSÃO}

No presente estudo, a letalidade geral atribuída à infecção pelo CHIKV foi menor que $1 \%$. As análises sugeriram que as maiores chances de óbito por infecção pelo CHIKV ocorreramm entre homens, com idade maior ou igual a 60 anos. A presença de manifestações clínicas como vômito e artrite, além de comorbidades como diabetes, hipertensão arterial, doença renal crônica e hepatopatia também se mostraram associadas ao desfecho. O conhecimento das comorbidades e manifestações clínicas específicas da infecção pelo $\mathrm{CHIKV}$ pode auxiliar o diagnóstico correto garantindo o manejo adequado e oportuno. A existência de agregados espaciais de casos e óbitos em cidades do Estado de Pernambuco, aponta para a necessidade de identificação de áreas com transmissão, de populações sob risco de adoecimento e planejamento das estratégias de vigilância e controle.

3. Simo FBN, Bigna JJ, Well EA, Kenmoe S, Sado FBY, Weaver SC, et al. Chikungunya virus infection prevalence in Africa: a contemporaneous systematic review and meta-analysis. Public Health. 2019; 166(1):79-88.

4. Wimalasiri-Yapa BMCR, Stassen L, Huang $X$, Hafner LM, Hu W, Devine GJ, et al. Chikungunya virus in AsiaPacific: a systematic review. Emerging Microbes and Infections. 2019; 8(1):70-9. 
5. Freitas ARR, Alarcón-Elbal PM, Donalisio MR. Excess mortality in Guadeloupe and Martinique, islands of the French West Indies, during the chikungunya epidemic of 2014. Epidemiology and Infection. 2018; 146(16):2059_ 65.

6. Leta S, Beyene TJ, De Clercq EM, Amenu K, Kraemer MUG, Revie CW. Global risk mapping for major diseases transmitted by Aedes aegypti and Aedes albopictus. Int J Infect Dis. 2018;67(1):25-35.

7. Huits R, De Kort J, Van Den Berg R, Chong L, Tsoumanis $A$, Eggermont $K$, et al. Chikungunya virus infection in Aruba: Diagnosis, clinical features and predictors of post-chikungunya chronic polyarthralgia. PLoS One. 2018; 13(4).

8. Simião $A R$, Barreto $F K$ de $A$, Oliveira R de MAB, Cavalcante JW, Neto ASL, Barbosa RB, et al. A major chikungunya epidemic with high mortality in northeastern Brazil. Rev da Sociedade Brasileira de Medicina Tropical. 2019;52:e20190266.

9. Silva Junior GB, Pinto JR, Mota RMS, Da Justa Pires Neto R, De Francesco Daher E. Risk factors for death among patients with Chikungunya virus infection during the outbreak in northeast Brazil, 2016-2017. Transactions of the Royal Society of Tropical Medicine and Hygiene. 2019; 113(4):221-226.

10. Paixão ES, Rodrigues LC, Costa M da CN, Itaparica M, Barreto F, Gérardin P, et al. Chikungunya chronic disease: A systematic review and meta-analysis. Transactions of the Royal Society of Tropical Medicine and Hygiene. 2018;112(7):301-316.

11. Hossain MS, Hasan MM, Islam MS, Islam S, Mozaffor M, Khan MAS, et al. Chikungunya outbreak (2017) in Bangladesh: Clinical profile, economic impact and quality of life during the acute phase of the disease. PLoS Neglected Tropical Diseases. 2018;12(6).

12. Brito CAA, Teixeira MG. Increased number of deaths during a chikungunya epidemic in Pernambuco, Brazil. Memorias do Instituto Oswaldo Cruz. 2017; 112(9):650651.

13. Brito CAA. Alert: Severe cases and deaths associated with Chikungunya in Brazil. Rev da Sociedade Brasileira de Medicina Tropical. 2017; 50(5):585-589.

14. Freitas ARR, Cavalcanti L, Von Zuben AP, Donalisio MR. Excess Mortality Related to Chikungunya Epidemics in the Context of Co-circulation of Other Arboviruses in Brazil. PLoS currents. 2017; 9(13):1-10.

15. Ministério da Saúde (MS). Secretaria de Vigilância em Saúde. Monitoramento dos casos de dengue, febre de chikungunya e febre pelo vírus Zika até a Semana Epidemiológica 52, 2016. Brasília: MS; 2017. 11 p.
16. Ministério da Saúde (MS). Secretaria de Vigilância em Saúde. Guia de Vigilância em Saúde. Brasília: MS; 2019. $740 \mathrm{p}$.

17. Costa S da SB, Branco MDRFC, Aquino Junior J, Rodrigues ZMR, Queiroz RC de S, Araujo AS, et al. Spatial analysis of probable cases of dengue fever, chikungunya fever and zika virus infections in Maranhao State, Brazil. Revista do Instituto de Medicina Tropical de Sao Paulo. 2018 Oct 25;60:e62.

18. Ministério da Saúde (MS). Secretaria de Vigilância em Saúde. Protocolo de investigação de óbitos por Arbovírus urbanos no Brasil - dengue, chikungunya e Zika. Brasília: MS; 2016. 35 p.

19. Freitas ARR, Gérardin P, Kassar L, Donalisio MR. Excess deaths associated with the 2014 chikungunya epidemic in Jamaica. Pathogens and Global Health. 2019; 113(1):27-31.

20. Dorléans F, Hoen B, Najioullah F, Herrmann-Storck C, Schepers KM, Abel $S$, et al. Outbreak of chikungunya in the French caribbean islands of martinique and guadeloupe: Findings from a hospital-Based surveillance system (2013-2015). Am J Trop Med Hyg. 2018; 98(6):1819-25.

21. Godaert L, Bartholet S, Dorléans F, Najioullah F, Colas S, Fanon JL, et al. Prognostic factors of inhospital death in elderly patients: A time-to-event analysis of a cohort study in Martinique (French West Indies). BMJ Open. 2018; 8(1).

22. Crosby L, Perreau C, Madeux B, Cossic J, Armand $C$, Herrmann-Storke $C$, et al. Severe manifestations of chikungunya virus in critically ill patients during the 2013-2014 Caribbean outbreak. Int J Infect Dis. 2016 Jul 1;48:78-80.

23. Vairo F, Haider N, Kock R, Ntoumi F, Ippolito G, Zumla A. Chikungunya: Epidemiology, Pathogenesis, Clinical Features, Management, and Prevention. Infectious Disease Clinics of North America. 2019; 33(4):1003-25.

24. Hsu CH, Cruz-Lopez F, Vargas Torres D, Perez-Padilla J, Lorenzi OD, Rivera A, et al. Risk factors for hospitalization of patients with chikungunya virus infection at sentinel hospitals in Puerto Rico. PLoS Negl Trop Dis. 2019;13(1).

25. Badawi A, Ryoo SG, Vasileva D, Yaghoubi S. Prevalence of chronic comorbidities in chikungunya: A systematic review and meta-analysis. Int J Infect Dis. 2018; 67(1):107-113.

26. Almeida BFK, Montenegro RM, Fernandes VO, Oliveira $R$, De Araújo Batista LA, Hussain A, et al. Chikungunya and diabetes, what do we know? Vol. 10, Diabetology and Metabolic Syndrome. BioMed Central Ltd.; 2018. 


\section{MONTE et al.}

27. Bonifay T, Douine M, Bonnefoy C, Hurpeau B, Nacher M, Djossou F, et al. Poverty and Arbovirus Outbreaks: When Chikungunya Virus Hits More Precarious Populations Than Dengue Virus in French Guiana. Open Forum Infect Dis. 2017; 4(4).

28. Fred A, Fianu A, Béral M, Guernier V, Sissoko D, Méchain $M$, et al. Individual and contextual risk factors for chikungunya virus infection: The SEROCHIK cross-sectional population-based study. Vol. 146, Epidemiology and Infection. Cambridge University Press; 2018. p. 1056-64.

29. Oviedo-Pastrana M, Méndez N, Mattar S, Arrieta G, Gomezcaceres L. Lessons learned of emerging Chikungunya virus in two populations of social vulnerability of the Colombian tropics: Epidemiological analysis. Arch Public Heal. 2018; 76(1).
30. Rossi G, Karki S, Smith RL, Brown WM, Ruiz MOH. The spread of mosquito-borne viruses in modern times: A spatio-temporal analysis of dengue and chikungunya. Spat Spatiotemporal Epidemiol. 2018; 26(1):113-25.

\section{CORRESPONDÊNCIA}

Ana Cristina Pedrosa do Monte

Rua Luís Cesário de Melo, 261, bloco C, apartamento 2

Casa Amarela, Recife, Pernambuco

Brasil, CEP 52081-010

E-mail: acpm19@gmail.com 